\title{
Vergleich der Eignung von vier Methoden (Immunfluoreszenz antinukleärer Antikörper, indirekte Hämagglutination, Immundiffusion, Gegenstromimmunelektrophorese) zur Differenzierung und Identifizierung von nukleäres Antigen
}

\author{
Von H.-J. Lakomek, N. Sablotni \\ Medizinische Klinik und Poliklinik C
}

H.-J. Hagedorn

Institut für Medizinische Mikrobiologie und Virologie und

\section{H. L. Krüskemper}

Medizinische Klinik und Poliklinik C der Universität Düsseldorf

(Eingegangen am 21. Februar/17. Mai 1983)

Zusammenfassung: Die Eignung von 4 Methoden (Immunfluoreszenz antinukleärer Antikörper (ANA), indirekte Hämagglutination, Immundiffusion und Gegenstromimmunelektrophorese) zum Nachweis und zur Differenzierung von Antikörpern gegen extrahierbares nukleäres Antigen (ENA) in Seren von 197 Patienten mit entzündlich rheumatischen Erkrankungen wurde analysiert. Die Gegenstromimmunelektrophorese zeigte die höchste Sensitivität und Spezifität für die Erfassung und Differenzierung von Antikörpern gegen Ribonucleoprotein (RNP), nụkleäres Glykoprotein (Sm) und Antikörpern, die bei Sicca-(Sjögren)-Syndrom (SS-B, Ha) beschrieben wurden. Diese Methode ist wie die Immundiffusion geeignet, die immunologische Identität von ENA-Antikörperseren zu Referenziseren darzustellen. Der Nachweis der immunologischen Identität in der Gegenstromimmunelektrophorese wurde mit einem neuentwickelten Testsystem (Pool-Verfahren) durchgeführt. Diese Methode zeigt im Vergleich zum Verfahren nach Kurata \& Tan ((1976) Arthritis Rheum. 19, 574-580) Vorteile hinsichtlich der Identifizierbarkeit und Praktikabilität.

Evaluation of 4 methods (immuñofluorescence of antinucleic antibodies (ANA), indirect haemagglutination, immunodiffusion, counterimmunoelectrophoresis) for the differentiation and identification of antibodies against extractable nucleic antigen (ENA)

Summary: The performance of four methods (immunofluorescence of antinucleic antibodies (ANA), indirect haemagglutination, immunodiffusion and counterimmunoelectrophoresis) in the detection and differentiation of the antibodies against extractable nucleic antigen (ENA) was analysed with the aid of sera from 197 patients suffering from inflammatory diseases. Counterimmunoelectrophoresis showed the highest sensitivity and specifity for the detection and differentiation of antibodies against ribonucleoproteins (RNP), nuclear glycoprotein (Sm) and antibodies, which occur in Sicca (Sjögren) syndrome (SS-B, Ha). This method, like immunodiffusion, is suitable for demonstrating the common immunological identity of the ENA-antibody sera and the reference sera, The proof of immunological identity using the counterimmunoelectrophoresis was performed with the newly developed test system (pool procedure). This method, as compared to the procedure of Kurata \& Tan ((1976) Arthritis Rheum. 19, 574-580) has certain technical advantages, and gives better identification. 


\section{Einfïhrung}

Für entzündlich rheumatische Erkrankungen scheinen Antigen-Antikörper-Systeme nicht nur eine beträchtliche diagnostische Relevanz zu haben, sondern zudem eine Bedeutung für die Einschätzung der Prognose und des Krankheitsverlaufes. Zur Charakterisierung und Unterscheidung von Kernantigenen wird deren Empfindlichkeit gegenüber nukleolytischen und proteolytischen Enzymen sowie Hitze herangezogen. Zum anderen wird die Krankheitsspezifität des entsprechenden Antikörpers beurteilt. So ist der n-DNA-Antikörper auf Grund seiner Inzidenz von $70 \%$ (1) in Sera von Patienten mit systemischem Lupus erythematodes eine wichtige diagnostische Kenngröße, wobei seine Konzentration mit der Krankheitsaktivität eng korreliert (2-5). Weitere Kernantikörper, die bei systemischem Lupus erythematodes im Serum gefunden werden, sind der Desoxyribonucleoprotein (DNP)- und der Sm-Antikörper. Letzterer ist nahezu ausschließlich beim systemischem Lupus "erythematodes nachweisbar und wird daher als „Marker“-Antikörper gewertet (1, 6, 7). Ribonucleoprotein (RNP)-Antikörper finden sich dagegen bei verschiedenen entzündlich rheumàtischen Erkrankungen. Besonders hohe Titer werden in Sera von Patienten mit ,Mixed Connective Tissue Disease" gemessen $(1,8,9,10)$. Alspaugh und Mitarbeiter (11) fanden eine hohe Inzidenz von SS-A-Antikörpern (70\%) und SS-B-Antikörpern $(48 \%)$ in Sera von Patienten mit Sicca-Syndrom. Ebenfalls beim Sicca-Syndrom und auch beim systemischem Lupus erythematodes beschrieben Akizuki und Mitarbeiter (12) den Ha-Antikörper. Nach Meinung dieser Autoren sind der Ha- und der SS-B-Antikörper immunologisch identisch. Tan und Mitarbeiter (13) berichteten kürzlich über den Nachweis des Scl-70-Antikörpers, eines „Marker“-Antikörpers für die progressive systemische Sklerodermie.

Die genannten Antikörper können mittels der Immunfluoreszenz auf Gewebeschnitten erfaßt werden, eine Unterscheidung der Antikörperspezifitäten ist aber nur bedingt möglich.

So bewirkt der Desoxyribonucleoprotein (DNP)Antikörper an Zellkernen ein homogenes Muster in der Immunfluoreszenz, der nDNA-Antikörper dagegen ein ringförmiges (,rim“, oder „peripheral") Immunfluoreszenzmuster $(14,15)$. Ein fleckförmiges (,speckled") Fluoreszenzbild wird von Antikörpern gegen verschiedene lösliche Kernantigene (ENA-Antikörper) hervorgerufen (Tab. 1). Zu diesen gehören der RNP-, der Sm-, der SS-B-, der Haund der Scl-70-Antikörper $(6,11,12,13,16)$. Zur Differenzierung der für das „speckled“ Immunfluo- reszenzmuster verantwortlichen Antikörpèr ist ein Extrakt aus Kaninchen- oder Kalbsthymuszellkernen (ENA-Extrakt) $(9,17)$ geeignet, der die Antigene dieser fünf Antikörper enthält (15). In den letzten Jahren wurden unter Verwendung dieser.Extrakte Methoden zum Nachweis der genannten Antikörper entwickelt, u. a. eine Doppeldiffusionsmethode in Agarosegel nach Ouchterlony $(6,11,12,13$, 16) die indirekte Hämagglutination $(1,9,18)$ sowie die Gegenstromimmunelektrophorese $(17,19,20)$. Eine weitere Möglichkeit zur Erfassung von RNPAntikörpern bietet eine Modifikation der Immunfluoreszenzmethode, die antinukleäre AntikörperRibonuclease (ANA-RNase)-Technik $(10,21)$.

Die vorliegende Arbeit vergleicht diese vier Methoden zum Nachweis von Antikörpern gegen Thymuszellkernextrakt (ENA-Antikörpern) und versucht, die einzelnen Verfahren zu werten hinsichtlich

1. der Möglichkeit, mehrere Antigen-AntikörpērSysteme auf einmal zu erfassen (Differenzierbarkeit)

\section{2. der Identifizierbarkeit der Antikörper}

3. der Empfindlichkeit der Methoden

4. Ihrer Praktikabilität für die Anwendung in einem Routinelabor,

5. einer Strategie für den Einsatz dieser vier Methoden in einem Routinelabor.

\section{Material und Methoden}

Es wurden Sera von 197 Patienten mit entzündlich rheumatischen Erkrankungen untersucht: systemischer Lupus erythematodes (n $=46)$; "Overlap"-Syndrom $(n=14)$; progressive systemische Sklerodermie $(n=48)$; Spondylitis ankylosans $(n=36)$; rheumatoide Arthritis $(n=50)$; Verdacht auf Immunerkiankung $(n=3)$; außerdem Sera von Kontrollpersonen $(n=23)$. Die Sera wurden bis zur Untersuchung eingefroren $\left(-20^{\circ} \mathrm{C}\right)$ und vor dem Test de= komplementiert $\left(56^{\circ} \mathrm{C} / 30 \mathrm{~min}\right)$.

\section{Diagnosen}

Patienten mit systemischem Lupus erythematodes und rheumatoider Arthritis erfüllten die jeweiligen Klassifikationskriterien der American Rheumatism Association (22, 23). Die Diagnose progressive systemische Sklerodermie wurde gestellt, wenn typische Hautveränderungen und eine Organbeteiligung vorlagen (24). Ein „Overlap"-Syndrom wurde diagnostiziert bei gleichzeitigem Auftreten von mindestens zwei der folgenden Krankheitsbilder: systemischer Lupus erythematodes, progressive systemische Sklerodermie; Dermato-Polymyositis, Sjögren-Syndrom. Patienten mit Spondylitis ankylosans entsprachen den von Moll \& Wright (25) beschriebenen klinischen und radiologischen Charakteristika. Kontrollpersonen waren gesunde Klínikangestellte (Alter unter $40 \mathrm{~J}$.). 


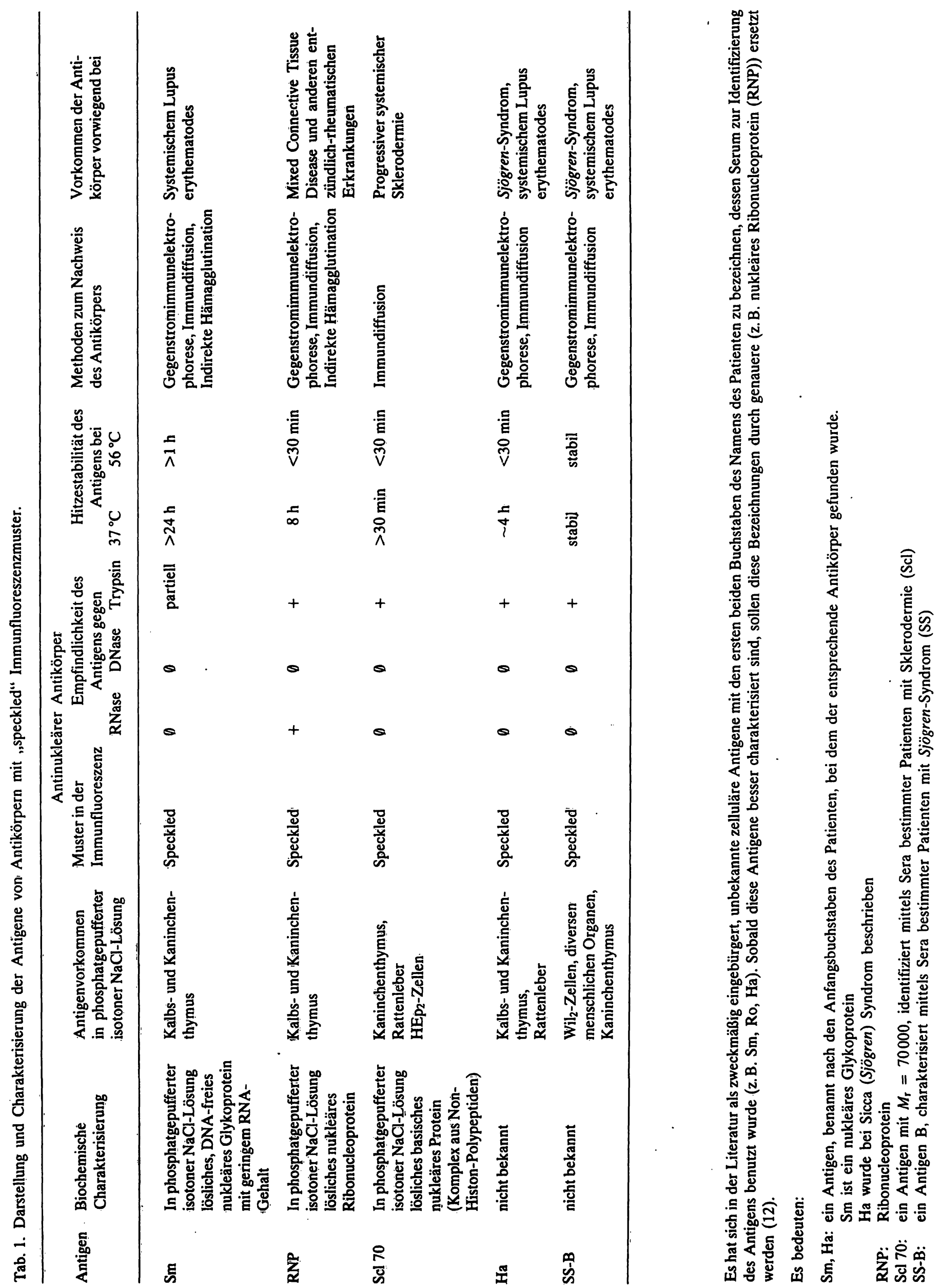




\section{Referenzsera}

Referenzsera mit Antikörpern gegen das RNP-, Sm- und SS-B-/ Ha-Antigen, die mit den von Dr. E. M. Tan benutzten identisch sind, wurden uns freundlicherweise vom Center for Disease Control, Atlanta, USA, zur Verfügung gestellt. Das Serum MZI wurde von E. S. Maxwell \& T. E. Martin, University of Chicago, Department of Biology, als anti-RNP-, das Serum AGR als antiRNP- und anti-Sm-haltig charakterisiert (persönliche Mitteilung). Beide dienten ebenfalls als Vergleichssera.

\section{Extrakt und Extraktbehandlung}

Als Antigenquelle diente ein lyophilisierter Extrakt aus Kaninchenthymus (Pel-Freez-Biologicals, Rogers, Arkansas).

Zur Antigendifferenzierung wurde der Extrakt mit Ribonuclease (RNase) (Serva Feinbiochemica, Heidelberg), Desoxyribonuclease (DNase) (Worthington Biochemical Corporation, Freehold, New Jersey) oder Trypsin (Serva Feinbiochemica, Heidelberg) inkubiert. Die RNase- und DNase-Behandlung erfolgte nach Kurata \& Tan (17), die Trypsinbehandlung nach Keiser \& Weinstein (20), wobei hier nach der Inkubation dem Enzym-Substrat-Gemisch eine der Trypsinaktivität entsprechende Menge an Trypsininhibitor (Serva Feinbiochemica, Heidelberg) zugesetzt wurde. Die Hitzebehandlung des Kaninchenthymusextraktes erfolgte bei $56^{\circ} \mathrm{C}$ für $30 \mathrm{~min}$.

Alle Patientensera wurden in mehreren Testansätzen ạn verschiedenen Tagen mit jedem der folgenden Verfahren untersucht.

\section{Indirekte Hämagglutination}

Für den Hämagglutinationstest wandten wir eine Modifikation der Methode von Sharp und Mitarbeitern an (9). Eine Suspension gewaschener defibrinierter Schafserythrocyten $(40 \mathrm{ml} / 1)$ in Veronalpuffer ( $\mathrm{pH} 7,4$ ) mit einem Tanningehalt von $25 \mathrm{mg} / \mathrm{l}$ wurde 30 min bei Raumtemperatur inkubiert und anschließend einmal in Veronalpuffer gewaschen. Aus dem Sediment wurde eine neue Suspension $(330 \mathrm{ml} / 1)$ hergestellt und mit gleichen Volumina des unbehandelten bzw. vorbehandelten Kaninchenthymusextrakt (Proteingehalt $10 \mathrm{~g} /$ ) 30 min bei Raumtemperatur inkubiert. Danach wurde viermal mit Veronalpuffer, der normales Kaninchenserum (Behringwerke Marburg) im Verhältnis 1:150 enthielt, gewaschen. Die beladenen Erythrocyten wurden in kaninchenserumhaltigem Puffer so resuspendiert, daß die Suspension bei einer Wellenlänge von $578 \mathrm{~nm}$ und einer Schichtdicke von $1 \mathrm{~cm}$ eine Transmission von $24 \%$ ergab.

Zur Absorption unspezifischer Antikörper wurden die Testsera zunächst mit gleichen Volumina eines nicht antigenbeladenen Erythrocytenkonzentrates bei $37^{\circ} \mathrm{C}$ für $30 \mathrm{~min}$ inkubiert. Nach Zentrifugation wurden die absorbierten Sera in Zehnerschritten mit kaninchenserumhaltigem Puffer verdünnt und jeweils $50 \mu \mathrm{l}$ auf Mikrotiterplatten (Flow Laboratories, Meckenheim) übertragen. Nach Zugabe von $50 \mu \mathrm{l}$ der Gebrauchssuspension mit sensibilisierten Erythrocyten erfolgte die Inkubation der Testansätze über Nacht bei $40^{\circ} \mathrm{C}$. Das Testergebnis wurde als positiv gewertet, wenn in einer Serumverdünnung von mindestens 1:100 ein deutliches Agglutinationsbild zu erkennen war. Ein Abfall des mit indirekter Hämagglutination ermittelten Titers bei Verwendung von RNase-behandeltem Kaninchenthymusextrakt um mindestens zwei Titerstufen sprach für das Vorliegen eines RNP-Antikörpers, nach RNase-Inkubation bestehende Resttiter $\geqslant 1: 100$ zeigten Sm-Antikörper an.

\section{Immundiffusion}

Die Immundiffusion wurde durchgeführt wie von Dorsch und Mitarbeitern (26) beschrieben. 0,75 g Agarose L (Behringwerke Marburg) wurde in $100 \mathrm{ml}$ phosphat-gepufferter isotoner $\mathrm{NaCl}$ Lösung $(0,15 \mathrm{~mol} / 1$; $\mathrm{pH} \mathrm{7,2)}$ gelöst und je $12 \mathrm{ml}$ davon auf Glasplatten $(8,3 \mathrm{~cm} \times 9,3 \mathrm{~cm})$ gegossen. In das èrkaltete Gel wurden
Löcher für Serum bzw. Antigen gestanzt mit einem Durchmesser von $4 \mathrm{~mm}$ und einem Abstand zwischen Antigen- und Antikörperloch von $3 \mathrm{~mm}$. Die Protein-Konzentration des Kaninchenthymus-Extraktes betrug $20 \mathrm{~g} / 1$ Puffer, die Sera wurden unverdünnt sowie verdünnt eingesetzt. Die Platten wurden 4 Tage in der feuchten Kammer bei $40^{\circ} \mathrm{C}$ inkubiert, anschließend in physiologischer $\mathrm{NaCl}$-Lösung gewaschen und mit Coomassie-Blue gefärbt.

\section{Gegenstromimmunelektrophorese}

Die Elektrophorese erfolgte nach der Methode von Kurata \& Tan (17). 0,6 $\mathrm{g}$ Agarose $\mathrm{H}$ (LKB, Bromma, Schwedenn) wurde in $100 \mathrm{ml} \mathrm{1:1} \mathrm{mit} \mathrm{destilliertem} \mathrm{Wasser} \mathrm{verdünntem} \mathrm{Michaelispuffer}$ $(0,025 \mathrm{~mol} / 1 ; \mathrm{pH} 8,2)$ gelöst, und je $10 \mathrm{ml}$ davon auf Gläsp̄lătten $(8,3 \mathrm{~cm} \times 9,3 \mathrm{~cm})$. gegossen. In das erkaltete Gel wurdeñ zwei parallele Lochreihen gestanżt (Dürchmesser $4 \mathrm{~mm}$; Reihenabstand $10 \mathrm{~mm}$ ). In die kathodennahe Lochreihe wurde unbehandelter oder vorbehandelter Kaninchenthymus-Extrakt der Protein-Konzentration $10 \mathrm{~g} / \mathrm{Puffer}$ gegeben. Unverdünñtes oder verdünntes Test- und Referenzserum wurde in die anodennahe Reihe gefüllt. Die Durchführung der Gegenstromimmunelektínophiorese erfolgte mit einem Multiphor -Elektrophoresesystem (LKB, Bromma, Schweden) bei einer konstanten Spannung von $10 \mathrm{~V} / \mathrm{cm}$ über 1 Stunde. Die Plattèn wưrden über Nacht in der feuchten Kammer inkubiert, anschließend mït Natriumcitratlösung ( $50 \mathrm{~g} / \mathbf{1})$ gewaschen und mit Coomassie-Blue gefäribt.

Immunfluoreszenzmethoden

(Antinukleärer Antikörper/Antinukleärer Aṇtikörp̣er-RNase)

Für die Bestimmung des Titers und Fluoreszenzmusters der Anti= nukleären Antikörper (ANA) in der indirekten Immunfluoreszenz wurden Rattenleberschnitte (Hylland-Travenol, München) verwendet. Die RNase-Vorbehandlung des Antigensubstrates erfolgte nach der von Rosenthal angegebenen Methode, wobei die Organschnitte mit RNase (Serva Feinbiochemica, Heidelberg) der Konzentration $0,1 \mathrm{~g} / \mathrm{l}$ während $30 \mathrm{~min}$ bei $37^{\circ} \mathrm{C}$ inkubièrt wurden. Ein Serum wurde als RNP-Antikörper-haltig gewertet, wenn es bei einem "speckled" Fluoreszenzmuster und einem Ausgangstiter von 1:1280 oder mehr einen Titerabfall auf RNase-behandelten Schnitten von mindestens drẹi Stufen zeigte (21).

\section{Ergebnisse}

Der Vergleich der Antinukleären Antikörper (ANA)-Immunfluorèsżenztiter mit den Antikörperbefunden in der Hämagglutination, der Immundiffusion und der Gegenstromimmunelektrophorese ergibt, daß sich bei 76 Sera mit einem Immunfluoreszenz-Titer von $\geqslant 1: 320$ in 25 Fällen ( $32 \%$ ), bei 144 Sera mit einem ANA-Titer $<1: 320$ oder negativem Immunfluoreszenz-Test dagegen nur zwẹimal $(1,4 \%)$ Antikörper gegen Thymusextrakt mit mindestens einer Methode fanden (Abb. 1). Eine Assoziation zwischen einem hohen ImmunfluoreszenżTiter und einer bestimmten Antikörperspezifität konnte hier nicht beobachtet werden.

Die K̇riterien von Rosenthal zum Ribonucleoprotein (RNP)-Antikörpernachweis (Immunfluoreszenz-Titer von $\geqslant 1: 1280$, "speckled" Immunfluoreszenzmuster, Titerabfall um mindestens 3 Stufen auf RNase=behandelten Organschnitten) erfüllten 10 Sera (Tab. 2). 


\section{Viele Gründe}

sprechen für das System

\section{nach Maß:}

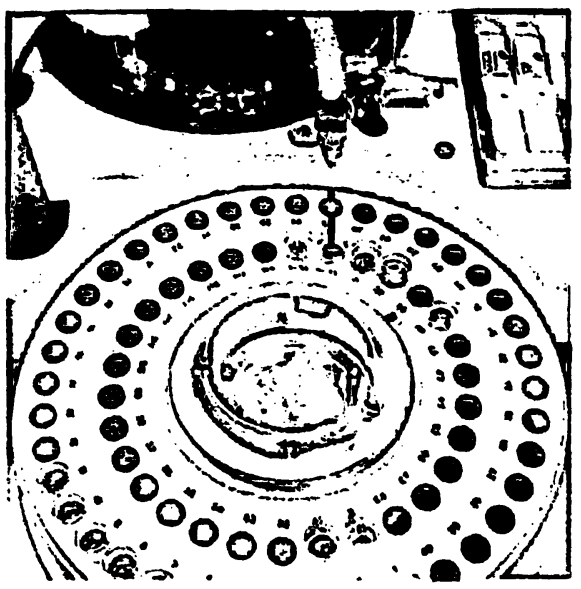

Routine, Eilanalysen mit Elektrolyten

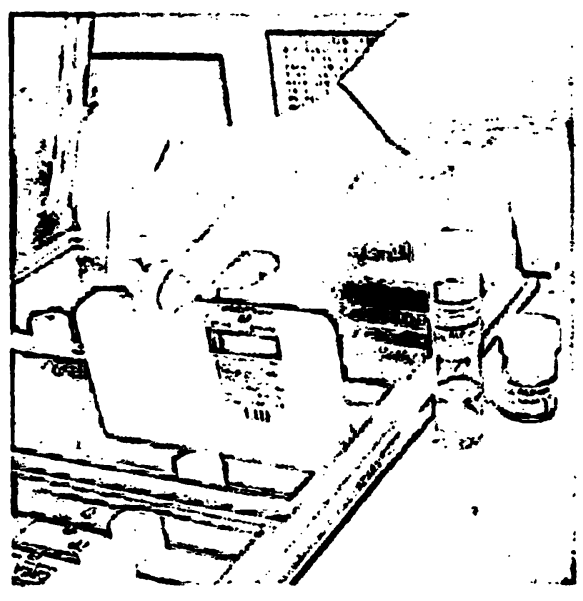

Maßgerechte

Packungsvarianten

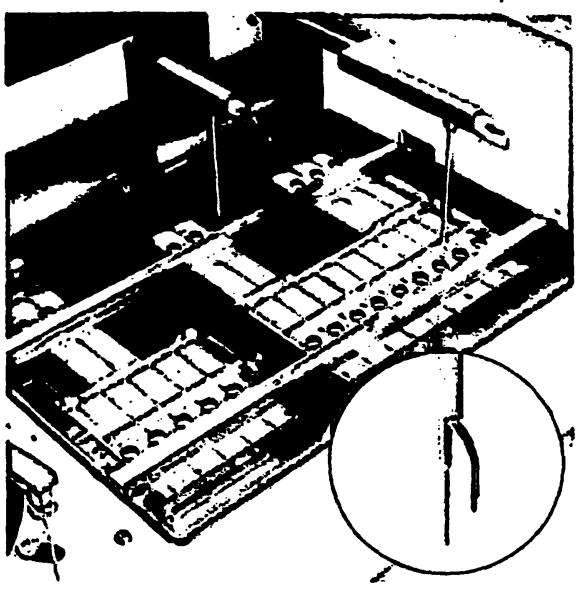

Sensorkontrolliertes Pipettiersystem

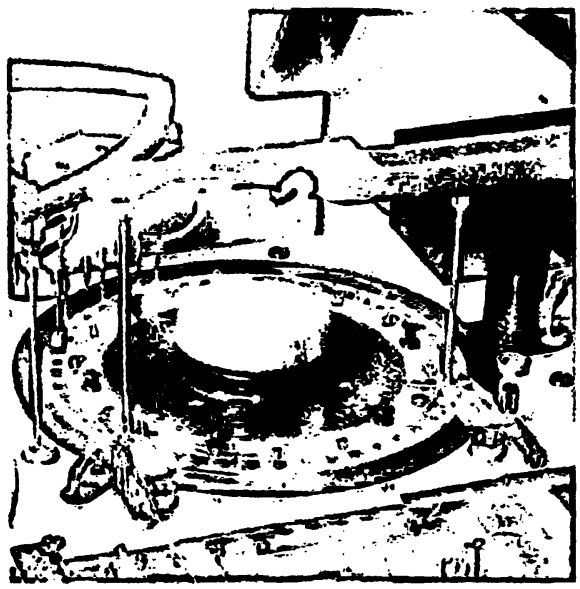

Reaktionsrotor

mit 48 Meßküvetten

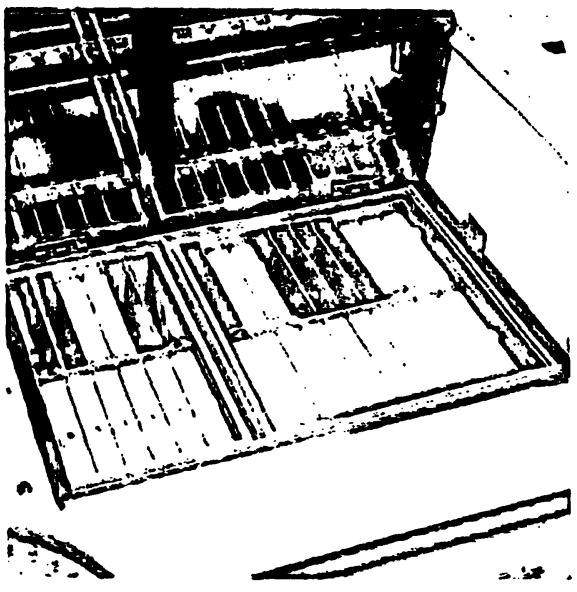

Integrierte Kühleinheit für Reagenzien

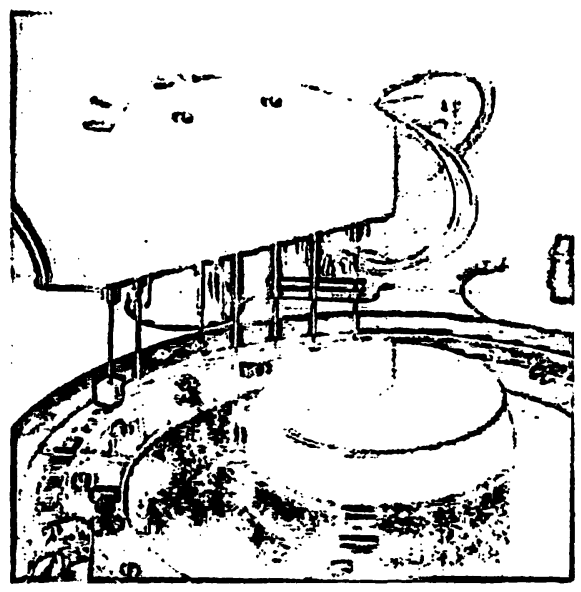

Wascheinheit mit integriertem automatischem Küvettenabgleich

HITACHI 705 -

die anspruchsvolle

Gerätetechnik

mit der hohen

Wirtschaftlichkeit

Bitte um Aufnahme in

Ihren Hitachi-Infoservice

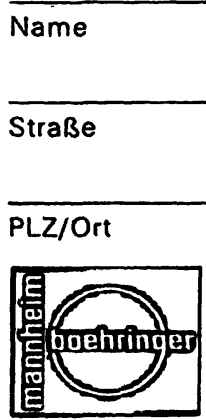

Boehringer Mannheim Gmbl Abt. M-DPG

Postfach 310120

6800 Mannheim 31 


\section{Walter de Gruyter Berlin-New York}

\section{K. Fotherby S. B. Pal (Editors)}

\section{购. K. Aganwal} (Editor)

\section{Hormones in Normal and Abnormal Human Tissues. \\ Volume 1}

1980. $17 \mathrm{~cm} \times 24 \mathrm{~cm} . \mathrm{XIV}, 658$ pages with figures and tables.

Hardcover. DM 145,-; apprōx. US \$69.25

ISBN 3110080311

Volume 2

$1981.17 \mathrm{~cm} \times 24 \mathrm{~cm}$. XII, 552 pages with figures and tables.

Hardcover. DM 135,-; approx. US \$64.50

ISBN 3110085410

\section{Volume 3}

$1982.17 \mathrm{~cm} \times 24 \mathrm{~cm}$. Approx. 500 pages with figures and tables.

Hardcover. Approx. DM 120,-; approx. US \$57.25

ISBN 3110086166

Hormones in Normal and Abnormal Human Tissues is a threevolume monograph dealing with the circulating levels, the pathological and therapeutic conditions and the factors controlling the secretion of non-polypeptide, protein and steroid hormones.

An attempt has been made to place emphasis on the concentrātion of the various hormones in tissues; where they are produced and where they might localize and produce an effect, and how these levels are modified under various circumstances.

\section{Hormone Antagonists}

$1982.17 \mathrm{~cm} \times 24 \mathrm{~cm}$. IX, 734 pages. Numérous illustrations. Hardcover. DM 180,-; approx. US $\$ 85.75$ ISBN 3110086131

This book groups together under one single cover antagonists for those hormones where antagonism has been documented specifically and with a certain degree of certitude. The major emphasis has been delineation of anti-hormone activity at the level of the hormone receptor but other aspects, such as antibody mediated antagonism and inhibition of synthesis, have been included to indicate other possible levels of inhibition of hormone activity. Clinical aspects, too, have been covered where they were documented with certitude.

It is felt that the book represents a major new reference source for years to come. Scientists, medical academicians, and advanced graduate students may use the book as a departing point for further pursuit of their own field. Involved research workers will find the volume of much interest since it provides data not published elsewhere. The book may also be used as a text volume to indicate the diversity and the wealth of information on the subject of hormone antagonism both in the basic research and in clinical medicine. Photo-offset method of publication assures expediency before specialized articles obsolete novelty.

Prices are subject to change without notice. 


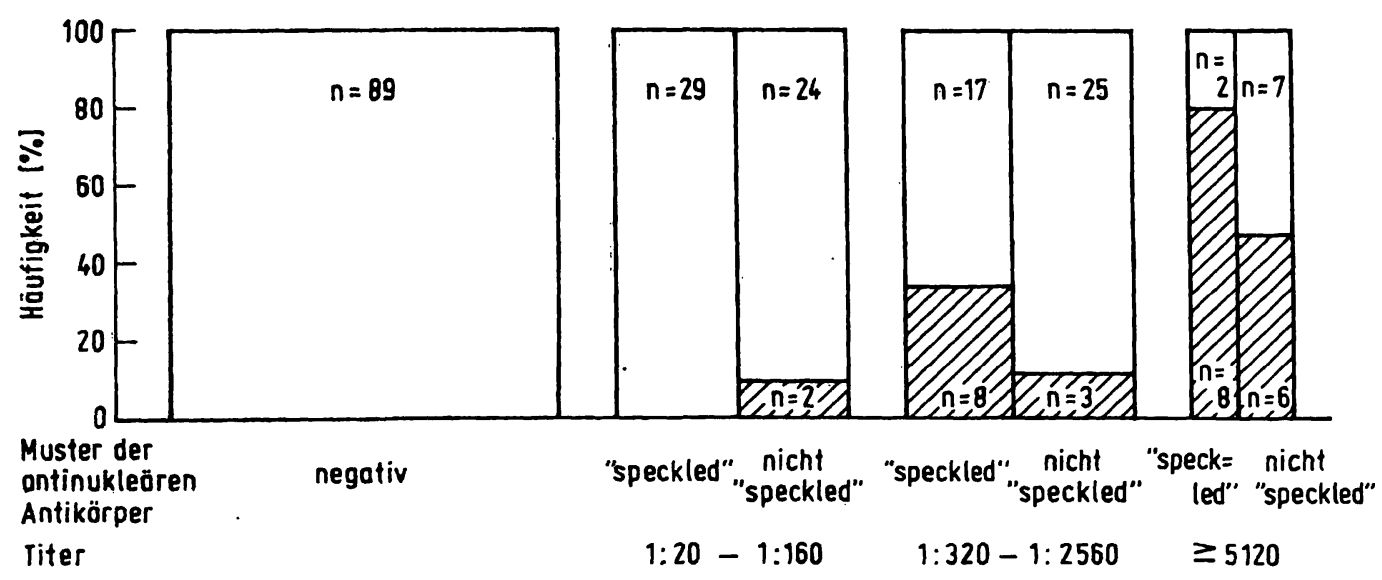

Abb. 1. Häufigkeit des Nachweises der Antikörper gegen Kaninchenthymus-Extrakt in Abhängigkeit von Titer und Fluoreszenzmuster. Offene Säulen: kein Nachweis; schraffiert: Nachweis mit mindestens einer Methode.

Die vergleichende Untersuchung dieser Sera in der Gegenstromimmunelektrophorese und der indirekten Hämagglutination zeigte aber, daß in zwei Fällen ausschließlich ein $\mathrm{Ha}$ - bzw. ein Sm-Antikörper nachgewiesen werden konnte.

Zwei weitere Sera waren negativ und zweimal konnten neben einem RNP-Antikörper zusätzlich noch andere Antikörperspezifitäten erfaßt werden. $\mathrm{Zu}$ dem fanden sich bei Anwendung der Elektrophorese oder Hämagglutination 9 RNP-Antikörpersera, die nicht die von Rosenthal geforderten Bedingungen erfüllten.

\section{Indirekte Hämagglutination}

Für die Test-Durchführung erwiesen sich in AlseverLösung suspendierte, 7 bis 10 Tage alte Schafserythrocyten als am besten geeignet. Es erfolgte eine Modifizierung der Enzymbehandlung, indem der Kaninchenthymus-Extrakt erst nach Inkubation mit RNase auf die Erythrocyten gegeben wurde. Dieses Vorgehen ergab identische Ergebnisse im Vèrgleich mit der von Sharp und Mitarbeitern (9) angegebenen Technik, in der die RNase-Behandlung erst nach Beladung der Erythrocyten mit dem Extrakt vorgenommen wird.

Von den in der Hämagglutination eingesetzten Testsera ergaben 13 (Immunfluoreszenz-Titer $\geqslant 1: 320$ ) einen positiven Reaktionsausfall mit Titern zwischen $1: 10^{2}$ und $1: 10^{6}$. Aufgrund des Reaktionsverhaltens vor und nach RNase-Behandlung des Kaninchenthymus-Extraktes enthielten 6 Sera einen RNP-, 4 einen Sm- und 3 weitere Sera einen RNP-sowie einen Sm-Antikörper (Tab. 2).

\section{Immundiffusion}

Von den in der Immundiffusion getesteten Sera ( $\mathbf{n}=$ 220) zeigten 17 eine Einzelbande, nur bei einem Testserum trat eine Doppelbande auf. In Abhängigkeit vom Verhalten der Sera gegenüber dem enzymund hitzebehandelten Extrakt konnten Antikörper gegen vier Kernantigene unterschieden werden (Tab. 2).

Von 11 in der Immundiffusion charakterisierten RNP-Antikörpersera zeigten 8 mit einem anti-RNP Referenzserum immunologische Identität, 2 Sera Nichtidentität und ein Serum partielle Identität. Ein anti-SS-B-Serum und 2 anti-Ha-Sera verhielten sich ebenfalls fraglich partiell identisch mit dem antiRNP-Serum. Somit war in 6 von 14 Fällen keine sichere Zuordnung der Sera zu einer Antikörperspezifität möglich.

\section{Gegenstromimmunelektrophorese}

Mit diesem Verfahren konnten die in Tabelle 2 aufgeführten Antikörperspezifitäten nachgewiesen werden. Die Identität der Antikörper mit den Referenzsera wurde in einem von uns für die Gegenstromimmunelektrophorese entwickelten Pool-Verfahren überprüft. Nebeneinander wurden an der Anodenseite der Elektrophoreseplatte aufgetragen:

1. das Referenzserum,

2. das zu testende Serum (beide 1:2 mit Puffer verdünnt),

3. eine Mischung aus gleichen Volumina des unverdünnten Test- und Referenzserums. 


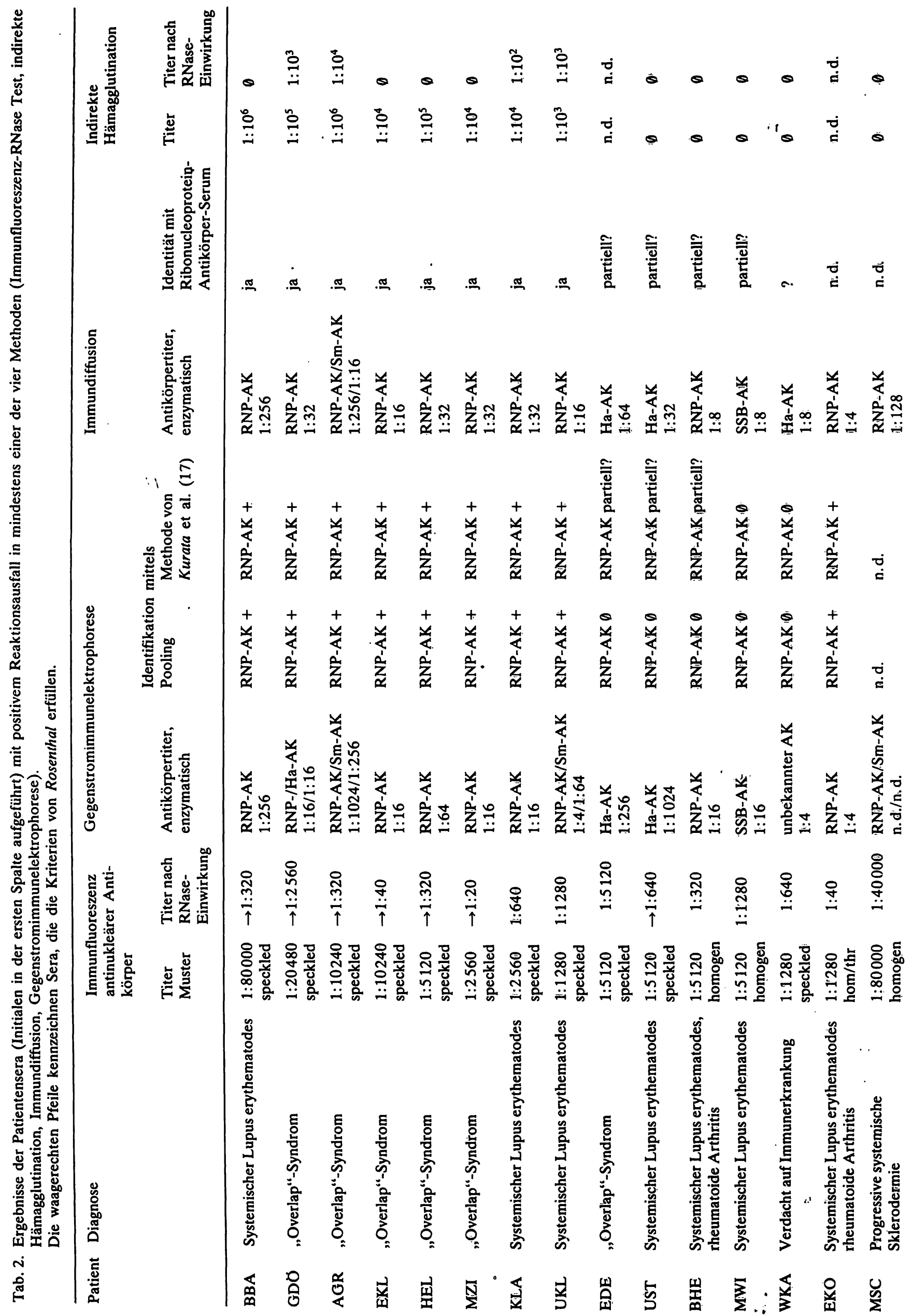




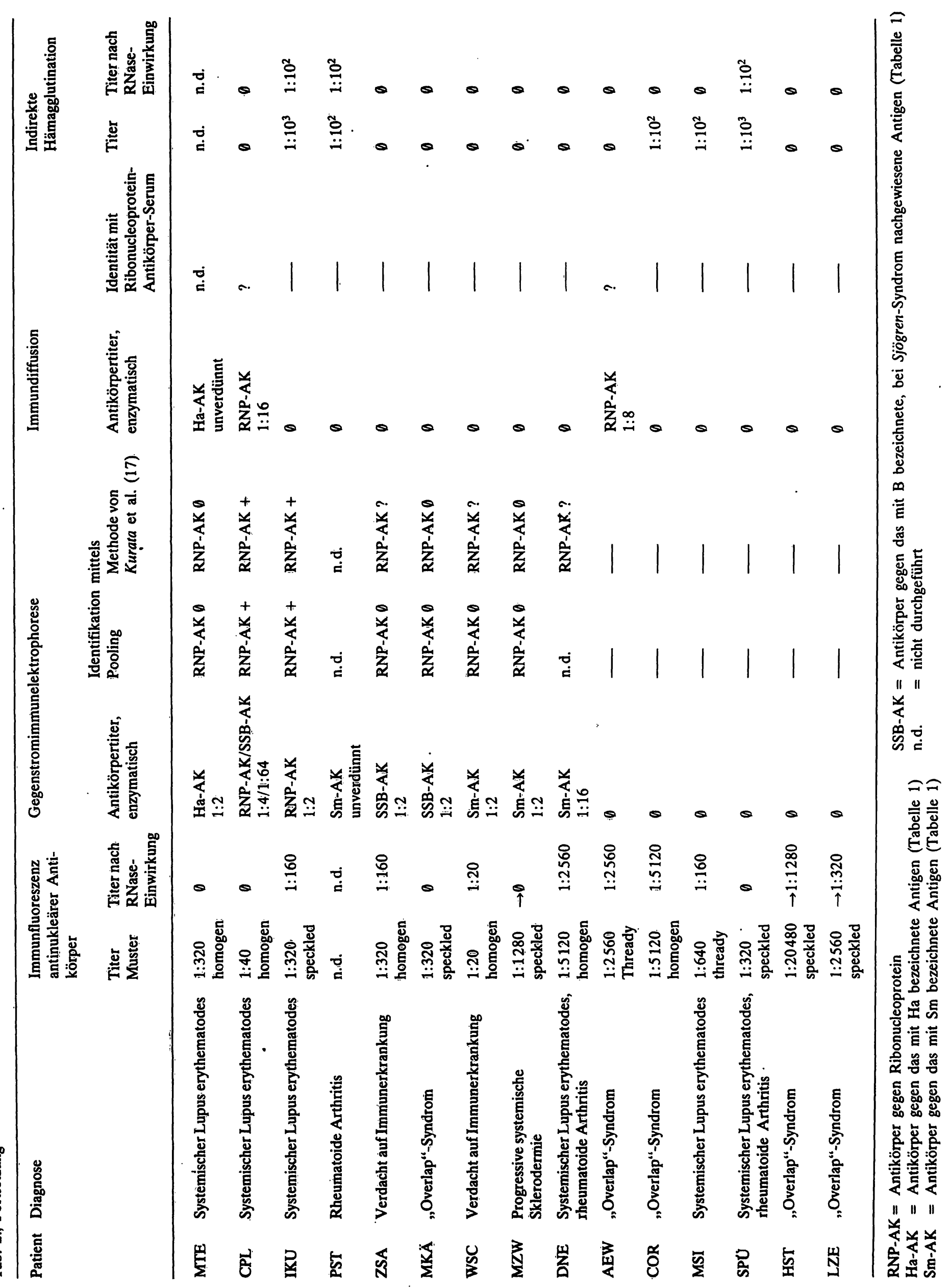


Alle einzeln aufgetragenen Sera zeigten Präzipitationsbanden. Trat im Vergleich zum Testserumansatz im Serumpool eine zusätzliche Bande auf, wurde dieser Befund als immunologische Nichtidentität mit dem Referenzserum gewertet. Fand sich im Serumpool nur eine Bande, wurde das Testresultat wie folgt kontrolliert:

1. das unverdünnte Referenzserum wurde mit unterschiedlichen Testserumverdünnungen zusammengegeben,

2. das unverdünnte Testserum wurde mit Verdünnungen des Referenzserum gepoolt.

Zeigte sich in einer der Kombinationen eine zusätzliche Bande, so sprach dies für immunologische Nichtidentität, anderenfalls wurde die Identität beider Antikörper angenommen (Abb. 2).

In 11 von 12 mittels der Elektrophorese charakterisierten anti-RNP-Sera ergab das Pool-Verfahren den Hinweis auf Identität mit dem anti-RNP-Referenzserum. Nur ein Serum zeigte Nichtidentität. Für die 9 monospezifischen Nicht-RNP-Antikörpersera bestätigte sich die Nichtidentität mit dem RNP-Antikörper.

Der Vergleich der Poolmethode mit der von Kurata \& Tan (17) angegebenen Elektrophorese-Technik der Antikörperidentifizierung ergab in 16 von 21 Fällen eine Úbereinstimmung beider Verfahren in der Abgrenzung von Identität und Nichtidentität

$\begin{gathered}\text { Kaninchenthymus- } \\ \text { Extrakt }\end{gathered}$
I III Antiserum gegen
SS-B (1:2)
II Serum HEL (1:2)
Ribonucleoprotein

Abb. 2. Identifikation von Antikörpern mittels des Pool-Verfahrens in der Gegenstromimmunelektrophorese: Die Identität des Antikörpers in Serum HEL mit dem RNP-Antikörper ist oben dargestellt (eine gemeinsame Bande der gepoolten Sera). Die Nichtidentität zum SSB-Antikörper ergibt sich aus dem Auftreten einer Doppelbande im Serumpool (unten). zum anti-RNP-Serum. Die übrigen 5 Sera zeigten im Pool-Verfahren klar Nichtidentität, während die Methode von Kurata \& Tan in diesen Fällen keine eindeutige Befundinterpretation zuließ. Dreimål schien eine partielle Identität mit dem anti-RNPReferenzserum vorzuliegen.

\section{Vergleich von Hämagglutination und Immundiffusion}

Nur bei den 7 Sera mit RNP-Antikörpertitern von mindestens 1:1000 im Hämagglutinationstest konnte auch in der Immundiffusion ein RNP-Antikörper nachgewiesen und die immunologische Identität mit dem anti-RNP-Referenzserum bestätigt werden. 2 RNP-Antikörpersera mit einem durch indirekte Hämagglutination ermittelten Titer von 1:100 präzipitierten dagegen nicht.

Von 7 mit der Hämagglutination gefundenen SmAntikörpersera zeigte nur eines (durch indirekte Hämagglutination ermittelter Titer nach RNase-Behandlung 1:10000) in der Immundiffusion eine mit dem anti-Sm-Referenzserum identische Präzipitationslinie. Die übrigen 6 Sera mit durch indirekte Hämagglutination ermittelten Resttitern von 1:100 oder 1:1000 reagierten in der Immundiffusion nicht wie Sm-Antikörpersera. Andererseits fand sich bei 6 im Hämagglutinationstest negativen Sera in der Immundiffusion eine Bande.

Während im Hämagglutinationstest nur zwei Antikörperspezifitäten unterschieden werden können, ermöglicht die Immundiffusion, Antikörper gegen mindestens vier Kernantigene żu differenzieren (RNP, Sm, SS-B, Ha). Der Vergleich beider Methoden ergab zudem, daß niedrigtitrige RNP- und SmAntikörper mit Hämagglutinationstest-Titern unter 1:1000 nur in der indirekten Hämagglutination erfaßt werden.

\section{Vergleich von Hämagglutination und Gegenstromimmunelektrophorese}

Alle 9 Sera mit einem Hämagglutinationstest-Titer $\geqslant 1: 1000$ bildeten Präzipitationsbanden, wobei die Antikörper-Titer in beidẹn Methoden eng miteinander korrelierten (Tab. 2). Von 4 Sera mit einem Hämagglutinationstest-Titer unter 1:1000 zeigte nur eines eine Reaktion in der Gegenstromimmunelektrophorese. Andererseits ermöglicht die Elektrophorese die Identifizierung von 11 Sera, die im Hämagglutinationstest negativ waṛẹn (Tab. 2). 
Vergleich von Immundiffusion und Gegenstromimmunelektrophorese

In der Immundiffusion und Gegenstromimmunelektrophorese konnten weitgehend identische Antikörpertiter gemessen werden; nur in 3 Sera fanden sich in der Elektrophorese wesentlich höhere Titer. Bemerkenswert ist aber, daß 7 Sera, die in der Elektrophorese Antikörpertiter unter 1:32 hatten, in der Immundiffusion nicht präzipitieren. Darüberhinaus konnten in 4 Sera, die in der Immundiffusion nur eine Bande zeigten, in der Gegenstromimmunelektrophorese Doppelbanden gesehen und somit zusätzlich weitere Antikörperspezifitäten erfaßt werden.

Nur ein Serum zeigte ein gegensätzliches Verhalten, d.h. dieses reagierte nur in der Immundiffusion (Tab. 2).

\section{Diskussion}

In der durchgeführten Studie konnte in 16 von 64 Sera $(25 \%)$ mit einem ,speckled" Fluoreszeñżmuster ein Antikörper gegen Kaninchenthymus-Extrakt gefunden werden. Ein vergleichbares Ergebnis wurde von Farber \& Bole (27) berichtet. Bedeutender als der Fluoreszenztyp scheint für den Nachweis von Antikörpern gegen Kaninchenthymus-Extrakt der Immunfluoreszenz-Titer zu sein, da in 25 von 76 Sera $(32 \%)$ mit einem Immunfluoreszenz-Titer von 1:320 oder höher ein Thymusextrakt-Antikörper unabhängig vom Fluoreszenzmuster gesehen wurde (Abb. 1). Obwohl in Sera mit einem Immunfluoreszenz-Titer von $\geqslant 1: 320$ und ,speckled" Fluoreszenzmuster die Häufigkeit der Antikörper gegen Kaninchenthymus-Extrakt maximal war $(16 / 35=$ $46 \%$ ), muß die Einbeziehung dieses Fluoreszenzmusters als Auswahlkriterium für den Einsatz von Hämagglutination, Immundiffusion und Gegenstromimmunelektrophorese kritisch gesehen werden, dà von den 27 Sera mit Antikörpern gegen Kaninchenthymusextrakt zwar 16 ein „speckled“ Muster in der Immunfluoreszenz aufweisen, sich aber auch in 11 Sera ein nicht=,,speckled" Muster findet. Tan (28) stellte dar, daß gleichzeitig vorhan= dene Kernantikörper mit „rim“ oder „,homogenem“ Fluoreszenzmuster das Mưster von Antikörpern gegen lösliche Kernantigene überdecken und diese somit dem Nachweis entgehen können. Die Berücksichtigung des Immunfluoreszenz-Titers von $\geqslant 1: 320$ erhält noch einen höheren Stellenwert dadurch, daß 25 von 27 anti-Thymus-Extrakt-Sera diese Bedingung erfüllen.
Der Nachweis von RNP-Antikörpern mit dem von Rosenthal (21) beschriebenem Immunfluoreszenzverfahren ist trotz der Praktikabilität dieser Methode nur bedingt möglich. Einerseits konnte nur in 6 von 10 Sera, die den Kriterien von Rosenthal genügten, der RNP-Antikörperbefund mit anderen Verfahren bestätigt werden, andererseits konnte aber mittels Gegenstromimmunelektrophorese und Hämagglutination ein RNP-Antikörper auch in 9 Sera nachgewiesen werden, die die Bedingungen von Rosenthal nicht erfüllten. RNP-Antikörper sind durch die Immunfluoreszenz-RNase-Technik allein nicht sicher identifizierbar. Auch Jonsson \& Norberg (29) berichteten über eine weniger gute Korrelation der Ergebnisse von Hämagglutination und Immunfluoreszenz-RNase-Test beim Nachweis von RNPAntikörpern.

Die Hämagglutination ist trotz einer im Vergleich zum Immunfluoreszenz-RNase-Test höheren Empfindlichkeit ebenfalls als Routineverfahren zum Nachweis von Antikörpern gegen Kaninchenthymus-Extràkt nicht gut geeignet. Die eigenen Erfahrungen wie die anderer Autoren zeigen, daß die Qualität des gesamten Testsystems entscheidend von dem Alter der jeweils verwendeten Erythrocyten abhängt (18). Auch Kozin \& Fowler (30) vertreten die Auffassung, daß auf Grund der technischen Probleme bei der Beladung der Erythrocyten die indirekte Hämagglutination für ein klinisches Routinelabor nicht geeignet ist. Mit diesem Verfahren ist es lediglich möglich, Antikörper zu unterscheiden, die mit der RNase-empfindlichen Fraktion des Kaninchenthymus-Extraktes (= RNP-Antikörper) bzw. mit der RNase-unempfindlichen Antigenkomponente (= Sm-Antikörper) reagieren. Der Nachweis von niedrigtitrigen RNP-Antikörpern ist dann nicht möglich, wenn gleichzeitig Sm-Antikörper mit höherem Titer vorhanden sind $(18,26)$.

Der Vergleich der Ergebnisse der Hämagglutination mit den beiden Präzipitationsverfahren zeigt Antikörper gegen Kaninchenthymus-Extrakt mittels Gegenstromimmunelektrophorese bzw. Immundiffusion in 12 Fällen, bei denen die Hämagglutination negativ ist, obwohl sie gegenüber den anderen Verfahren das empfindlichere Nachweissystem darstellt $(1,13,18,30)$. Das negative Resultat der $6 \mathrm{Ha} / \mathrm{SS}-$ B-Antikörpersera im Hämagglutinationstest (Tab. 2) ist dadurch bedingt, da $B$ die Hämagglutination diese Antikörper nicht erfaßt (14). Jonsson und Mitarbeiter (31) sowie Venables und Mitarbeiter (32) beschrieben RNP-Antikörper, die ebenfalls in der indirekten Hämagglutination nicht nachweisbar waren, wobei als Erklärungsmöglichkeit ein Nicht- 
haften des zugehörigen Antigens an der Erythrocytenmembran diskutiert wurde. Möglicherweise liegt in 4 RNP-Antikörpersera diese Antikörperspezifität vor. Eine Erklärung für die Nichterfassung der 4 Sm-Antikörpersera mittels der Hämagglutination fand sich nicht (Tab. 2).

Gegenüber der Hämagglutination haben beide Präzipitationsverfahren den entscheidenden Vorteil, da $\beta$ auf Grund des Einsatzes von Referenzsera die Identifizierung von Antikörperspezifitäten möglich ist.

Die Wertigkeit der Charakterisierung von Antikörperspezifitäten auf der Basis der unterschiedlichen Enzymempfindlichkeit der eingesetzten Antigensubstrate (Organschnitte bzw. Kaninchenthymus-Extrakt) ist hier durch den direkten Vergleich mit Referenzsera überprüfbar. Der Einsatz von Referenzsera für RNP-, Sm- und Ha/SS-B-Antikörper in einer der Präzipitationsmethoden ergab bei 16 antiKaninchenthymusextrakt positiven Sera eine Ubereinstimmung mit der enzymatischen Zuordnung, in 4 Fällen dagegen eine Diskrepanz. Diese Abweichungen und die Tatsache, daß das Scl-70-Antigen (13) und das Ha-Antigen (12) sowie das Antigen des PM-1-Antikörpers (33) die gleiche Enzym- und Hitzeempfindlichkeit aufweisen, geben dem Einsatż von Referenzsera, wie bereits von anderen Arbeitsgruppen $(14,26,34)$ hervorgehoben, eine besondere $\mathrm{Be}=$ deutung für die eindeutige Zuordnung der gefundenen Antikörper.

Vergleicht man die Praktikabilität beider Präzipitationsverfahren, so hat die Gegenstromimmunelektrophorese dadurch Vorteile, daß hier in der Regel eine Auswertung nach wenigen Stunden möglich ist, während ein Ergebnis, in der Immundiffusion erst nach einigen Tagen abgelesen werden kann.

$\mathrm{Da}$ die gemessenen Antikörpertiter in der Immundiffusion und der Gegenstromimmunelektrophorese weitgehend übereinstimmten, fanden wir in beiden Methoden die gleiche Empfindlichkeit für den Nachweis von Kaninchenthymusextrakt-Antikörpern. Obwohl mit der Immundiffusion und der Gegenstromimmunelektrophorese prinzipiell die gleichen Antikörperspezifitäten erfaßbar sind, konnte bei vier in der Immundiffusion als monospezifisch charakterisierten Sera mittels der Gegenstromimmunelektrophorese durch das Auftreten einer zweiten Bande ein weiterer Antikörper differenziert werden (Tab. 2).

Unter Berücksichtigung der von Stites (35) därgestellten Kriterien für die Beurteilung der immunölogischen Identität von Antikörpern in der Immundiffusion zeigen 4 Sera (identisch mit dem anti-Ha/
SS-B-Referenzserum) Präzipitationsbilder, bei denen eine partielle Identität mit dem anti-RNP-Referenzserum vorzuliegen scheint, Nichtidentität aber nicht sicher ausgeschlossen werden kann. In weiteren 2 Fällen konnte auf Grund zu schwacher Präzipitationslinien kène Identifizierung, vorgenommen werden.

Andererseits zeigten 8 Sera typische Bilder immunologischer Identität.

Bei der Auswertung der mit dèr Methode von Kura= ta \& Tan (17) erhaltenen Ergebnisse treten ähnliche Schwierigkeiten auf (20), da auch bei diesem Verfahren die Unterscheidung von partieller Identität und Nichtidentität nicht immer möglich ist und zu schwache Präzipitationsbanden ebenfalls eine klare Identifizierung verhindern (Abb. 3).

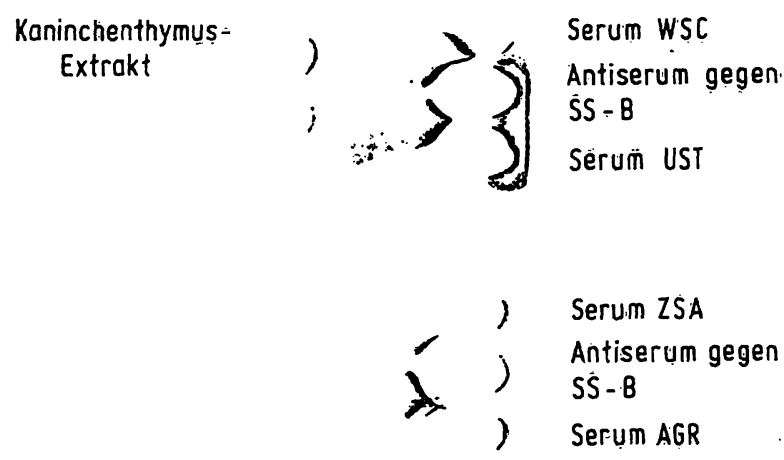

Abb. 3. Darstellung von Präzipitationsbildern im Identifikationsverfahren nach Kurata \& Tan am Bẹispiel des SS-BAntikörpers.

Serum UST zeigt Identität (konfluierende Bandenn), Serum AGR zeigt Nichtidentität (zwei nicht konflụierende Banden), Sera WSC und ZSA sind nicht sicher zuzuordnèn.

Die aufgezeigte Problematik der bekannten Verfahren in der Gegenstromimmunelektrophorese und der Immundiffusion führte zur Entwicklung einer neuen Referenżmethode für die Gegenstromimmunelektrophorese, die geeignet scheint, mittels eines Pools aus Test- und Referenzserum auch bei schwachen Banden oder fraglicher partieller Identität eine Zuordnung von Antikörpern zu ermöglichen. Zwar ist die Identifizierung von Testsera mit der Technik von Kuräta \& Tan weniger aufwendig, da das Poolverfahren aber offenbar Nichtidentität eindeutiger darstellt, sollte es gerade bei fraglichen Befunden zusätzlich Anwendung finden. Der kombinierte Einsatz beider Methoden stellt nach unserer Meinung eine Optimierung der Identifikationsmöglichkeiten in der Gegenstromimmunelektrophorese dar, weshalb auf der Basis der dargestellten Ërgebnisse dieses Vorgehen der Referenztechnik in der Immundiffusion vorzuziehen ist. .i 
Die vergleichende Untersuchung von Immunfluoreszenz-RNase-Technik, Hämagglutination, Immundiffusion und Gegenstromimmunelektrophorese führte zu der in Tabelle 3 unter vier Beurteilungskriterien aufgelisteten Bewertung. Zusammenfassend hat diese Studie gezeigt, da $B$ die Immunfluoreszenzmethode nach Rosenthal als Screening-Verfahren zum Nachweis von RNP-Antikörpern nicht geeignet ist, da insbesondere niedrigtitrige RNP-Antikörper hiermit nicht erfaßt werden. Die Hämagglutination hat auf Grund ihrer eingeschränkten Praktikabilität Nachteile für die Anwendung in einem Routinelabor, zudem werden auch hier RNP-Antikörper bei Maskierung durch gleichzeitig vorliegende höhertitrige Sm-Antikörper nicht erfaßt. Für die Gegenstromimmunelektrophorese und Immundiffusion wurde die Bedeutung der Anwendung von Referenzsera mit bekannter Antikörperspezifität betont. Durch das Pool-Verfahren ergibt sich ein praktikabler Ansatz, die Identifizierung von Anti- körpern gegen Kaninchenthymusextrakt ausschließlich mittels der Gegenstromimmunelektrophorese durchzuführen. Für die Erfassung und Differenzierung dieser Antikörper sind somit besonders die Immundiffusion und die Gegenstromimmunelektrophorese geeignet.

Auf der Grundlage der dargestellten Antikörpererfassung soll mittels Gegenstromimmunelektrophorese und Immundiffusion versucht werden, Beziehungen zwischen den serologischen Kenngrößen und der klinischen Symptomatik aufzuzeigen mit dem Ziel, die vielfältig beschriebenen Assoziationen von Kernantikörpern zu Diagnosen oder Krankheitscharakteristika der entzündlich rheumatischen Erkrankungen zu überprüfen.

\section{Danksagung}

Für die Uberlassung einiger Sklerodermieseren danken wir Herrn Dr. T. Krieg, Universitätshautklinik München.

Tab. 3. Vergleichende Darstellung von Immunfluoreszenz, indirekter Hämagglutination, Immundiffusion und Gegenstromimmunelektrophorese unter Anwendung von vier Beurteilungskriterien, wobei die Wertigkeit der Methoden von oben nach unten abnimmt.

\begin{tabular}{|c|c|c|c|}
\hline Differenzierbarkeit & Identifizierbarkeit & Empfindlichkeit & Praktikabilität \\
\hline $\begin{array}{l}\text { Gegenstromimmun- } \\
\text { elektrophorese }\end{array}$ & $\begin{array}{l}\text { Gegenstromimmun- } \\
\text { elektrophorese }\end{array}$ & Indirekte Hämagglutination & Gegenstromimmune \\
\hline Immundiffusion & Immundiffusion & Immunfluoreszenz & Immunfluoreszenz \\
\hline Indirekte Hämagglutination & - & $\begin{array}{l}\text { Gegenstromimmunelektrophorese/ } \\
\text { Immundiffusion }\end{array}$ & Immundiffusion \\
\hline
\end{tabular}

Immunfluoreszenz

Indirekte Hämagglutination/

Indirekte Hämagglutination

\section{Literatur}

1. Notman, D. D., Kurata, N. \& Tan, E. M. (1975) Ann. Intern. Med. 83, 464-469.

2. Adler, M. K., Baumgarten, A., Hecht, B. \& Siegel, N. J. (1975) Ann. Rheum. Dis. 34, 444-450.

3. Hughes, G. R. V. (1975) Scand. J. Rheumatol. Suppl. 11, $42-51$.

4. Pincus, T., Schur, P. H., Rose, J. A., Decker, J. L. \& Talal, N. (1969) N. Engl. J. Med. 281, 701-705.

5. Schur, P. H. \& Sandson, J. (1968) N. Engl. J. Med. 278, 533-538.

6. Tan, E. M. \& Kunkel, H. G. (1966) J. Immunol. 96, 464470.

7. Tan, E. M. (1967) J. Clin. Invest. 46, 735-745.

8. Parker, M. D. (1973) J. Lab. Clin. Med. 82, 769-775.

9. Sharp, G. C., Irvin, W. S., LaRoque, R. L., Velez, C., Daly, V., Kaiser, A. D. \& Holman, H. R. (1971) J. Clin. Invest. 50, 350-359.

10. Sharp, G. C., Irvin, W. S., Tan, E. M., Gould, R. G. \& Holman, H. R. (1972) Am. J. Med. 52, 148-159.

11. Alspaugh, M. A., Talal, N. \& Tan, E. M. (1976) Arthritis Rheum. 19, 216-222.

12. Akizuki, M., Powers, R. \& Holman, H. R. (1977) J. Clin. Invest. 59, 264-272.
13. Tan, E. M., Rodnan, G. R., Gárcia, I., Moroi, Y., Fritzler, M. J. \& Peebles, C. (1980) Arthritis Rheum. 23, 617-625.

14. Akizuki, M., Powers, R. \& Holman, H. R. (1977) Arthritis Rheum. 20, 693-701.

15. Nakamura, R. M. \& Tan, E. M. (1978) Hum. Pathol. 9, 8591.

16. Mattioli, M. \& Reichlin, M. (1971) J. Immunol. 107, 12811290.

17. Kurata, N. \& Tan, E. M. (1976) Arthritis Rheum. 19, 574580.

18. Nakamura, R. M., Peebles, C. L. \& Tan, E. M. (1978) Am. J. Clin. Pathol. 70, 800-807.

19. Bresnihan, B., Bunn, Ch., Snaith, M. L. \& Hughes, G. R. V. (1977) Br. Med. J. 1, 610-611.

20. Keiser, H. D. \& Weistein, J. (1980) Arthritis Rheum. 23, 1026-1035.

21. Rosenthal, M. (1977) Klin. Wochenschr. 55, 31-35.

22. Cohen, A. S., Reynolds, W. E., Franklin, E. C., Kulka, J. P., Ropes, M. W., Shulman, L. E. \& Wallace, S. L. (1971) Bull. Rheum. Dis. 21, 643-648.

23. Ropes, N. W., Bennett, G. A. \& Cobb, S. (1958) Bull. Rheum. Dis. 9, 175-180. 
24. Masi, A. T., Medsger, T. A., Rodnan, G. P., Fries, J. F., Altman, R. D., Brown, B. W., D'Angelo, W. A., LeRoy, E. C., MacKenzie, A. H., McShane, D. J. \& Sharp, G. C. (1979) Clinics in Rheumatic Diseases 5, 27-48.

25. Moll, J. M. H. \& Wright, V. (1973) Ann. Rheum. Dis. 32, 354-364.

26. Dorsch, C. A., White, G. M. \& Berzofsky, R. N. (1978) Am. J. Clin. Pathol. 71, 333-337.

27. Farber, S. J. \& Bole, G. G. (1976) Arch. İntern. Med. 136, 425-431.

28. Tan, E. M. (1967) J. Lab. Clin. Med. 70, 800-812.

29. Jonsson, J. \& Norberg, R. (1978) Scand. J. Rheumatol. 7, 229-236.
30. Kozin, F. \& Fowler, M. (1979) Am. J. Clin. Pathol. 71, 437440 .

31. Jonssón, J., Steiner, M. \& Ǩlein, E. (1976) Clin. Exp. Immunol. 25, 144-151.

32. Venables, P. J. W., Mumford, P. A. \& Maini, R. N. (1981) Ann. Rheum. Dis. 40, 217-223.

33. Wolfe, J. F., Adelstein, E. \& Sharp, G. C. (19777) J. Clin. Invest. 59, 176-178.

34. Northway, J. D. \& Tañ, E. M. (1972) Clin: Immunol. Im= munipathol. 1, 140-154.

35. Fudenberg, H. H., Stites, D. P., Caldwell, J. L. \& Well, J. V. (1980) Basic and Clinical Immunology, 343-381.

Dr. med. Heinz-Jürgen Lakomek Medizinische Klinik C

der Universität Düsseldorf

Moorenstr. 5

D-4000 Düsseldorf 